\title{
Interference Aware Full-Duplex MAC Protocols Design and Evaluation for the Next WLANs
}

\author{
Haifeng Luo*, Qian Jiang, Yong Liu, Huaixing Wang and Liang Huang \\ Department of Physics and Mechanical \& Electrical Engineering, \\ Hubei University of Education, Wuhan, 430205, China \\ 7935611@qq.com
}

\begin{abstract}
Recently the in-band full-duplex in wireless communication has evolved into a mature technology since the self-interference cancellation technique is able to support fullduplex capability. Moreover, some researchers focus on the full-duplex media access control (MAC) protocol design for wireless local area networks (WLANs). However, it is assumed that all the node have full-duplex capability in most study of the full-duplex $M A C$, and it is not practical since stations (STAs) may be half-duplex in the network while access point (AP) has full-duplex capability. In this paper, a full-duplex MAC protocol named interference aware full-duplex MAC (IAFM) is proposed for the next generation WLANs on the assumption only AP has full-duplex capability. In IAFM protocol, there are three mode including direct scheduling mode for normal full-duplex transmission, interference collection mode for interference collection usage and none full-duplex mode for legacy transmission. Beside, direct scheduling mode and interference collection mode are described in detail, and none full-duplex mode obeys the legacy MAC protocol in WLANs. The power control mechanism is also discuss in this paper. The simulation results shows that in saturation condition IAFM protocol with power control almost double the throughput of legacy MAC protocol in WLANs.
\end{abstract}

Keywords: WLANs, MAC, full-duplex, OFDMA

\section{Introduction}

The IEEE 802.11 wireless local area networks (WLANs) have been developed rapidly in the past two decades. The IEEE standard for WLANs was named as IEEE 802.4L at the beginning in 1988, while it was renamed as IEEE 802.11 in 1990 [1]. IEEE 802.11 standard defined the physical layer (PHY) and medium access control sub-layer (MAC) specification for the WLANs [2]. Moreover, all kinds of IEEE 802.11 task groups proposed many amendments to improve the IEEE 802.11 standard, and these amendments are integrated into IEEE 802.11 standard every few years. The latest complete version of IEEE 802.11 standard is the 2012 version. Similar to other communication networks, WLANs have evolved by introducing the latest technological advances in the wireless communication field as soon as they have become mature. For example, IEEE 802.11a in 1999 employed orthogonal frequency division multiplexing (OFDM), IEEE 802.11n in 2009 adopted single-user multiple input multiple output (SU-MIMO), aggregate MAC protocol data unit (A-MPDU), aggregate MAC service data unit (A-MSDU) and channel bonding, and IEEE 802.11ac in 2013 introduced downlink multi-user MIMO (MU-MIMO) transmissions. Furthermore, in the next generation WLANs named as IEEE 802.11ax which is in process and has no draft amendment uses a number of technologies including orthogonal frequency division multiple access (OFDMA) and uplink MU-MIMO to improve the efficiency of spectrum utilization [3]. Since there is more user data in the wireless networks [4], it is no doubt that more and more advanced and mature technologies will be introduced into WLANs. 
To further improve the network capability without using more bandwidth, in-band full-duplex capability, i.e., the ability of transmitting and receiving in the same band and at the same time, is propounded as a promising technology by researchers of wireless networks [5-7]. The main challenge for implementing full-duplex capability is that a node need to cancel the interference produced by its transmitting while it is receiving packet. Recent work [8-13] has demonstrated that the existing self-interference cancellation technology is mature enough to support full-duplex capability. To adopt full-duplex capability in WLANs could significantly improve the area throughput and increase spectral frequency reuse which is the goals of the next generation WLANs [14]. However, brand-new MAC protocols is needed for WLANs with full-duplex capability since the current MAC protocol of WLANs is designed for half-duplex communication networks.

There are totally two types full-duplex link, i.e., symmetric and asymmetric fullduplex link. Figure 1 shows these two types full-duplex link in WLANs. In symmetric full-duplex link access point transmits downlink packets to station and the receiving STA also transmits uplink packets to AP, while in asymmetric full-duplex link AP transmits downlink packets to STA2 and another STA, i.e., STA1 transmits uplink packets to AP. In addition, the interference between STAs (STA2 suffers from the interference due to the transmission of STA1) involved in full-duplex link could degrade the performance of full-duplex communication. Both AP and STAs should have full-duplex capability in symmetric full-duplex link, but only AP supports full-duplex capability and STAs may be half duplex nodes. So far some full-duplex MAC protocols are proposed on the assumption that all nodes have full-duplex capability [15-20]. However, there are a few full-duplex MAC protocols considering the scenario that AP supports full-duplex capability but STAs do not support full-duplex capability [21-23].

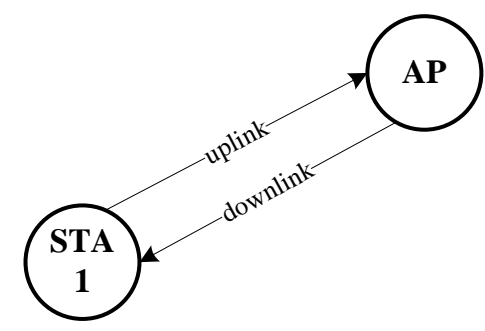

(a) symmetric full-duple link in WLANs

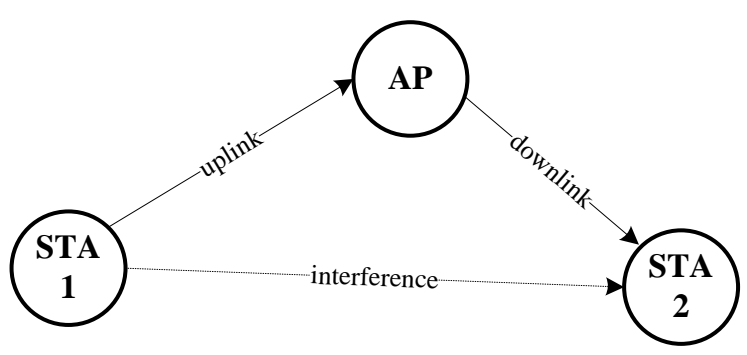

(b) asymmetric full-duple link in WLANs

\section{Figure 1. Symmetric and Asymmetric Full-Duplex Link in WLANs}

In this paper, the rest of the paper is organized as follows. In section 2, related work is introduced. The proposed full-duplex MAC protocol named IAFM is described in section 3. The simulation results are presented in section 4. And this paper is concluded in section 5 .

\section{Related Works}

\subsection{Full-duplex MAC Protocols}

So far, there are a lot of works focus on the full-duplex MAC design [15-22]. However, in most researchers assume that all the node in the network have full-duplex capability [15-19], and only a few researchers pay attention to study the full-duplex MAC protocols in which only AP has full-duplex capability and STAs have no full-duplex capability for WLANs [20-22]. In this paper, it is assumed that AP is a full-duplex node but STAs are all half-duplex nodes, and the co-existence between a full-duplex AP and half- 
duplex STAs should be considered. Thus, there are totally two links, i.e., the legacy link and the full-duplex link, in full-duplex MAC protocols. The legacy link is established by using the legacy MAC protocol described in IEEE 802.11, and the full-duplex link is set up by employing the full-duplex MAC protocol.

In [20] a full-duplex MAC protocol named as asymmetrical duplex (A-Duplex) is proposed. A-Duplex adopts capture effect to establish the full-duplex link, and the fullduplex link is established only if a STA accesses channel. Moreover, the mechanism of building the signal to interference (SIR) map is also introduce. However, the mechanism of collecting the interference is not very efficiency since it can only collect the interference of two STAs at a time and is not controlled by AP.

In [21], a contention based full-duplex MAC protocol is proposed. In this MAC protocol, when AP transmits a data frame to a STA, the other STAs, which do not affect the transmission of legacy link by using interference measurement mechanism, starts a secondary back-off procedure, and the winner of STA acquires the chance of using the fullduplex link.

In [22] it is proposed that after a STA wins the contention for channel access, AP announces some addresses of STA as the candidate for full-duplex link. And the candidate STAs start another back-off process to contend the full-duplex transmission opportunity. Moreover, [22] also introduces power control mechanism to improve the performance of proposed full-duplex MAC protocol.

\subsection{IEEE 802.11 MAC Protocol}

In WLANs, the MAC protocol is distributed coordinated function (DCF) based on carrier sensing multiple access with collision avoidance (CSMA/CA). In DCF, when a transmitter node (AP or STA) wants to send MAC protocol data units (MPDU) to the receiver node, it should first transmit a request to send (RTS) frame to the receiver node. Before transmitting the RTS frame, the transmitter node has to perform back-off process to avoid collisions. Each node chooses a random number within the range of 0 to the contention window as the value of back-off counter, and for every idle time slot the back-off counter decreases one. When the back-off counter is 0 , the node transmits the RTS frame and expects for the CTS frame. If the node that transmits RTS frames does not receive the clear to send (CTS) frame due to collisions, it need to double the contention window and performs back-off process again. At the first RTS transmission, the contention window of each node is the minimum contention window (i.e., CWmin), and it is doubled after collisions but is no more than the maximum contention window (i.e., CWmax).

After a transmitter node successfully transmits RTS frame, the receiver node needs to transmit CTS frame, and there are network allocation vector (NAV) duration in RTS and CTS frames for channel reservation. The nodes except transmitter node and receiver node overhearing the RTS and CTS frame acquire the NAV duration, and they cannot send any frames in the NAV duration. The transmitter node may send several MPDU or aggregate MAC protocol data unit (A-MPDU) frame in the reserved time, while the receiver replies acknowledgement (ACK) frame for MPDU frame or block acknowledgement (BA) frame. A-MPDU is a frame including a number of MPDU, and BA is able to acknowledge a block of MPDU by using bitmap. 


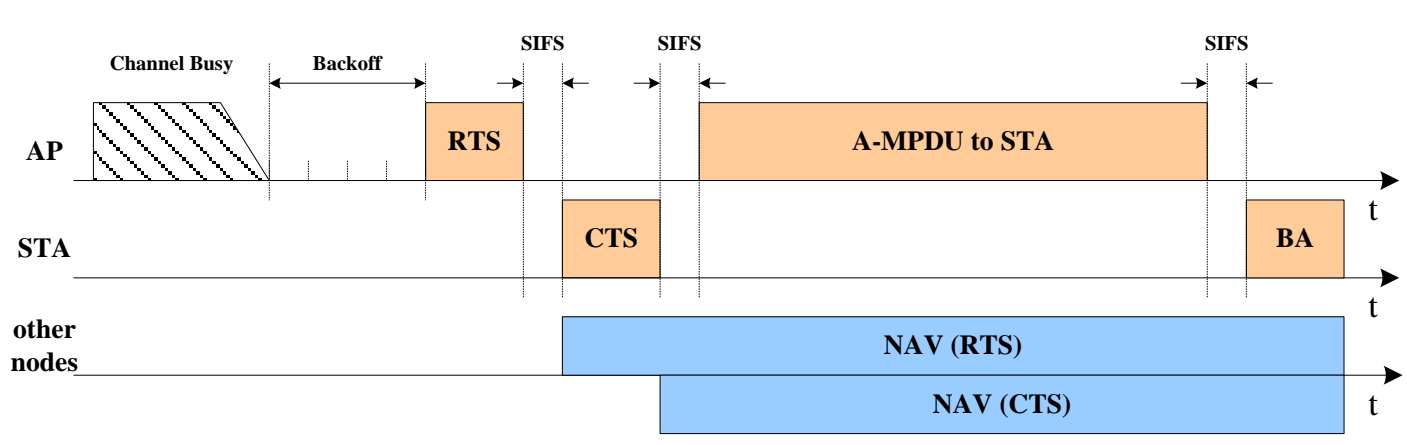

Figure 1. IEEE 802.11 MAC Protocol

\section{Protocol Design}

In this section, a full-duplex MAC protocol named as interference aware full-duplex MAC (IAFM) is proposed for WLANs. In IAFM, both AP and STA uses DCF to access channel for good compatibility with legacy WLANs, but the frame transmission part of IAFM is different and AP could set up a extra full-duplex link. When a node (AP or STA) wins the contention of channel access, there are three mode for IAFM protocol, i.e., direct scheduling mode, interference collection mode and none full-duplex mode. In IAFM with direct scheduling mode, AP directly selects a proper STA for full-duplex link based on the historical interference information, while in IAFM with interference collection mode, AP asks the STAs to report their interference state before selecting the STA in full-duplex link. The IAFM with none full-duplex mode is as the same as DCF with no full-duplex link. Some legacy fields in RTS and CTS frames are reused for establishing full-duplex link, and a new full-duplex field is added in the preamble of an AMPDU frame. Moreover, the perfect self-interference cancellation technology is assumed in the protocol designed of IAFM. Thus, AP does not suffer from the interference of its own transmission.

\subsection{IAFM with Direct Scheduling Mode}

Once AP has collect enough interference state information of STAs (the interference collection procedure is introduced in 3.2), AP is able to select a proper STA for the fullduplex link when a node (AP or STA) wins the contention. This kind of full-duplex transmission mode in IAFM is called direct scheduling mode. The frame transmission procedure of IAFM with direct scheduling mode is as similar as that in DCF, except that there is slight modification of RTS and CTS frame format. The downlink (AP wins the contention) transmission procedure and uplink (STA wins the contention) transmission procedure of IAFM with direct scheduling mode are shown in Figure 3 and Figure 4 respectively. Moreover, to simplify the description of IAFM the duration of busy channel and the back-off procedure is omitted in Figure 3 and Figure 4 (also in Figure 6 and Figure 7).

In Figure 3, after AP wins the contention, it send a full-duplex RTS (FD-RTS) frame to STA1. FD-RTS is a new frame for full-duplex link scheduling, and the comparison between RTS and FD-RTS is illustrated in Figure 5. AP uses full-duplex address field in the FD-RTS frame to announce the address of STA using full-duplex link. Specifically, AP selects a STA (STA2 in Figure 3) for full-duplex link based on historical interference information, and STA2 needs to send an A-MPDU frame to AP after receiving CTS frame. STA1 receives the FD-RTS frame, and then sends a CTS frame to AP after short inter-frame space (SIFS) obeying the inter-frame space rules in DCF. When AP receives CTS frame from STA1, it sends an A-MPDU frame to STA1 after SIFS. At the 
same time, STA2 sends an A-MPDU frame to AP. After the A-MPDU transmission, AP and STA1 simultaneously transmit BA frames as described in [21]. Moreover, it is easy for STA2 to determine the number of MPDU in the transmitting AMPDU frame, because the NAV duration minus 3 SIFS duration, a CTS transmission duration and a BA transmission duration is the period of the A-MPDU sent by AP.

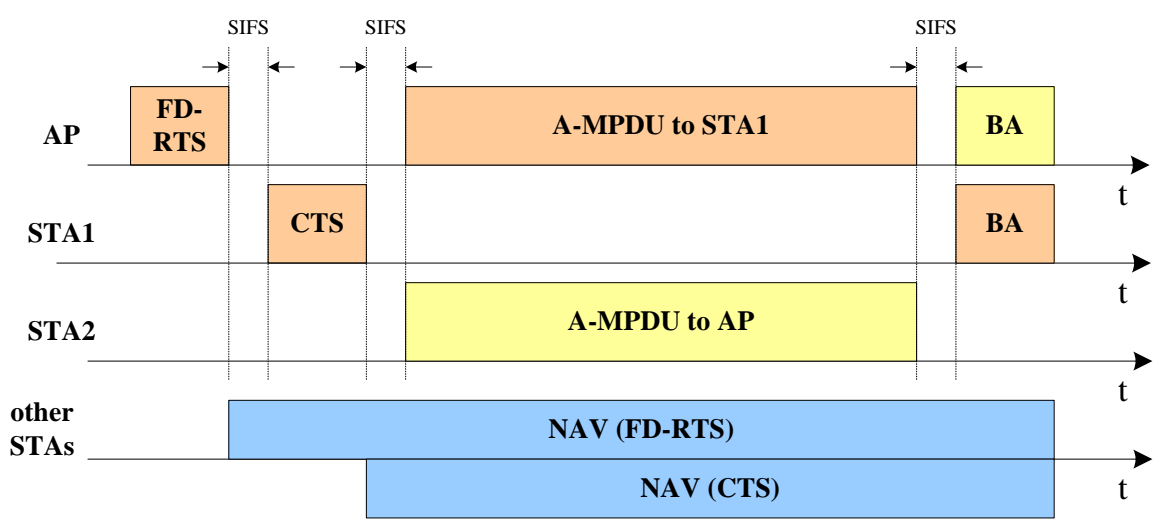

Figure 3. The Downlink Transmission Procedure of IAFM with Direct Scheduling Mode

As shown in Figure 4, STA1 sends an RTS frame to AP after the back-off procedure. Once AP receives the RTS frame, it sends a full-duplex CTS (FD-CTS) frame to STA1 and STA2 which is chosen by AP for full-duplex link based on historical interference information. There is also a full-duplex address field in FD-CTS as illustrated in Figure 5. STA1 receives the FD-CTS frame, and then transmits an A-MPDU frame to AP after SIFS. Meanwhile, AP transmits an A-MPDU to STA2. The two A-MPDU frames are received by AP and STA2 respectively. Then AP transmits a BA frame to STA1, and STA2 transmits a BA frame to AP.

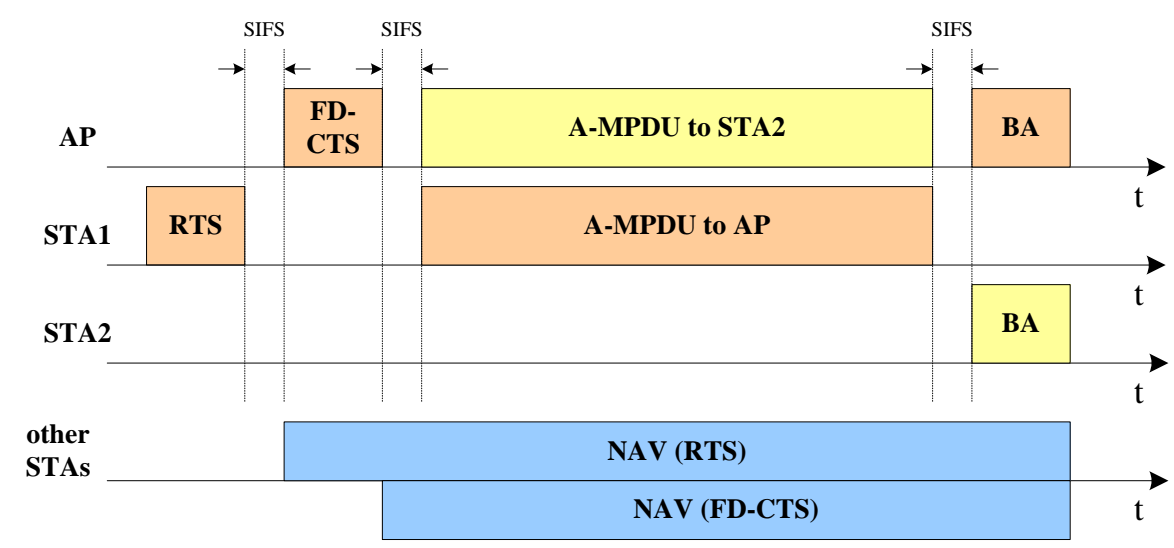

Figure 4. The Uplink Transmission Procedure of IAFM with Direct Scheduling Mode

The FD-RTS and FD-CTS frame format is illustrated in Figure 5. Compared with the legacy RTS and CTS frame, the receiver address field is replaced by full-duplex address field, and there are totally five sub-fields in full-duplex address field, i.e., FD mode, partial basic service set identifier (BSS ID), receiver association identifier (AID), FD AID and uplink TX power. The FD mode sub-field indicates the current mode of IAFM as 
described above including no full-duplex mode("00"), direct scheduling mode ("01"), interference collection mode ("10") and reserved mode ("11"), and the reserved mode is for future protocol extension. The partial BSS ID sub-field is the lower 12 bits of BSS ID, and it is used to make sure that the STAs receives the frame from the AP of their own BSS in multiple BSS scenario. The receiver AID sub-field is the AID of STA in legacy link, and the FD AID is the AID of STA in full-duplex link. The uplink TX power sub-field is adopted so that AP is able to control the power for uplink transmission (the power control issue is discussed in 3.3).

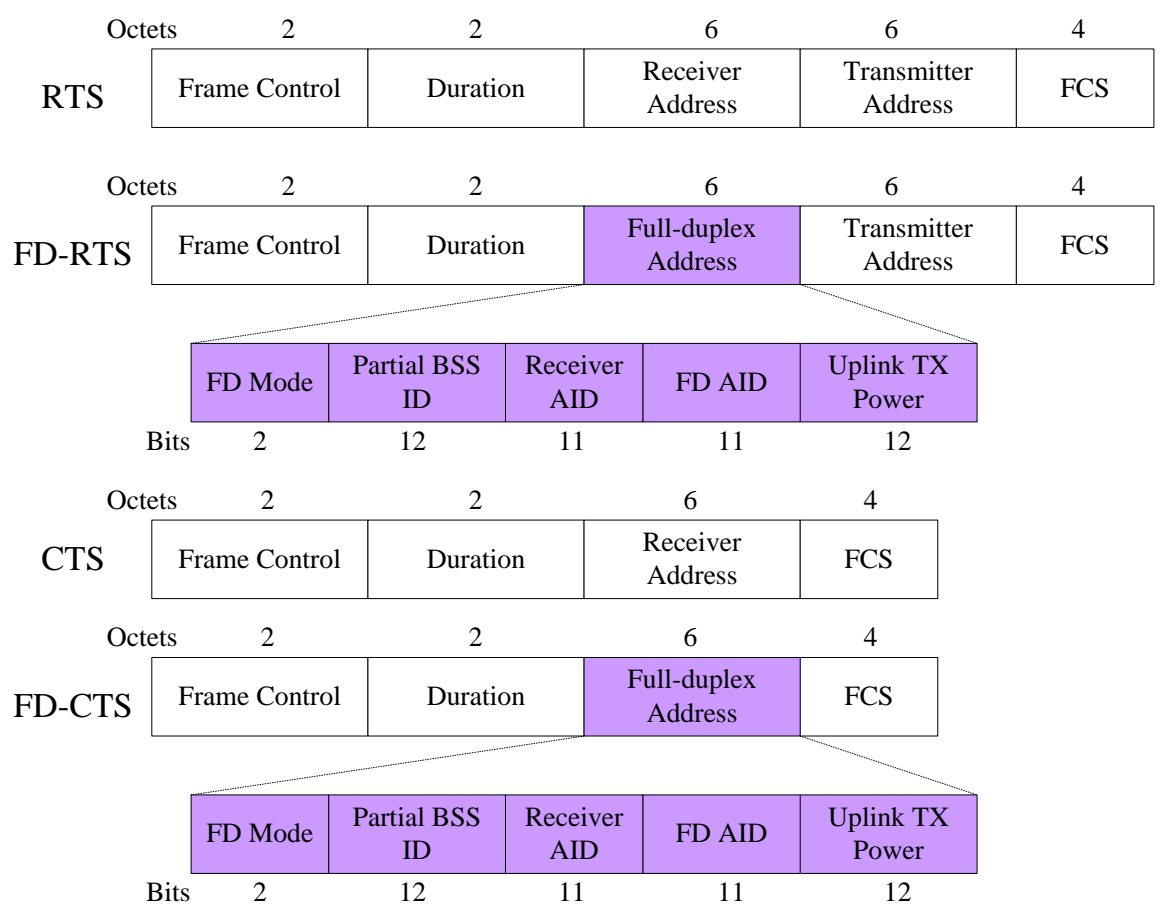

Figure 5. Frame Format of FD-RTS and FD-CTS

\subsection{IAFM with Interference Collection Mode}

It is obvious that the key problem of design a better full-duplex MAC protocol is to select a proper STA for full-duplex link. If AP has the interference state information between every two STAs, it is pretty easy for AP to schedule the proper STA involved in full-duplex link. Thus, the interference collection procedure is introduced in IAFM with interference collection mode. STAs including the STA in legacy link and candidate STA in full-duplex link all need to report their interference state in orthogonal frequency division multiple access (OFDMA) manner, and OFDMA is an important feature in the next generation WLANs [3]. In OFDMA technology the sub-carriers of the whole channel is divided into some sub-channels, and each sub-channel includes several sub-carriers. Multiple STAs is able to report their interference state simultaneously using OFDMA, and thus decrease signaling overhead.

Figure 6 illustrates the downlink transmission procedure of IAFM with interference collection mode. After AP and STA1 complete the exchange of FD-RTS (the FD mode sub-field in FD-RTS is interference collection mode) and CTS, AP transmits an interference state collection (ISC) frame to a number of STAs including STA1 and those candidate STAs for full-duplex link (STA2, STA3 and STA4). The frame of ISC contains $n$ receiver information fields ( $n$ is the number of sub-channels, and three are 4 subchannels in Figure 6) including the receiver AID and the sub-channel allocation subfield as shown in Figure 8. All the STAs in the same BSS need to measure the received 
power of FD-RTS and CTS, and those STAs who's AID is in the ISC frame transmit interference state report (ISR) frames to report their received power of the FD-RTS frame and the CTS frame. Moreover, STA1 only need to measure and report the received FD-RTS power if its AID is in the ISC frame. When AP receives all the ISR frames and acquires the interference state of STAs, it selects a STA for the full-duplex link, whose transmission does not cause the unsuccessful transmission of legacy link (the specific selection measure is introduced in 3.3). Then, AP transmits an A-MPDU to STA1 and announces the AID of STA in full-duplex link for STA3 and the uplink transmitting power for STA3 in FD preamble (FD-preamble is illustrated in Figure 8). STA3 receives the FD-preamble, and then transmits an A-MPDU to AP with the transmitting power indicated in FD-preamble. After the A-MPDU transmission, AP and STA1 transmit BA frames at the same time.

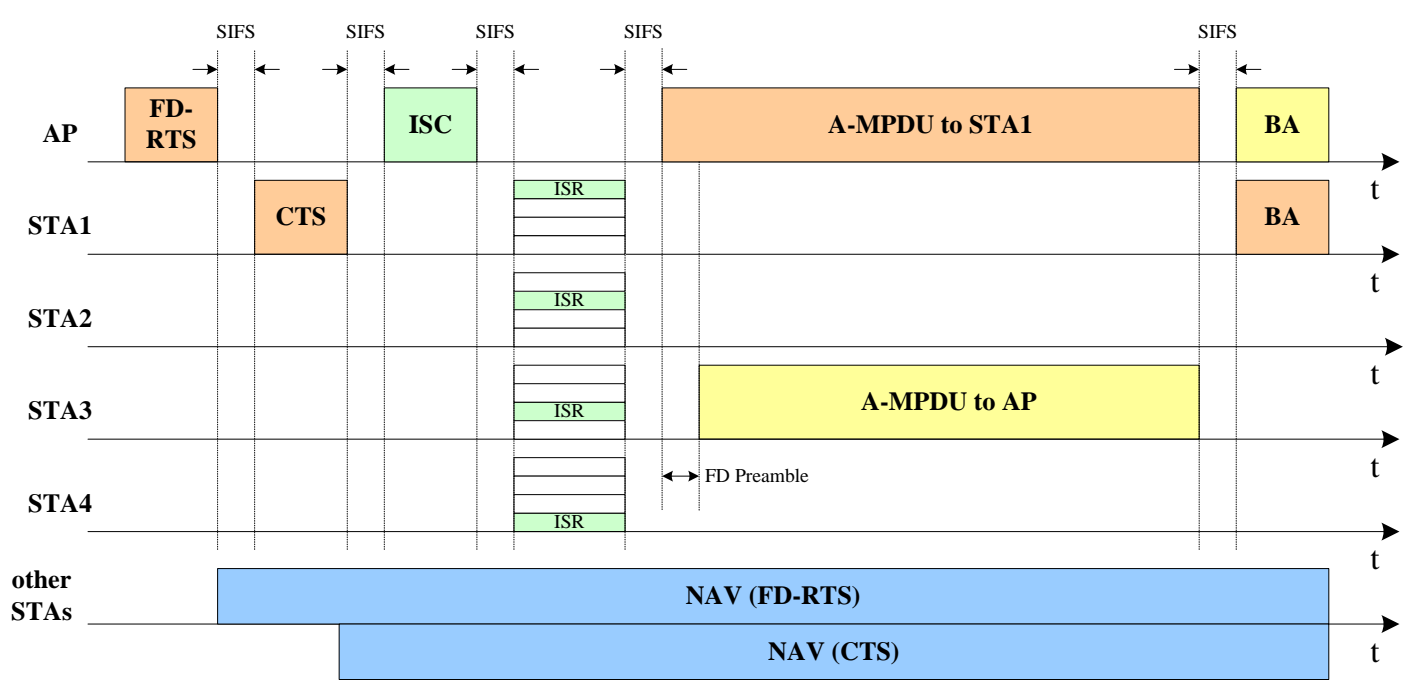

\section{Figure 6. The Downlink Transmission Procedure of IAFM with Interference Collection Mode}

The uplink transmission procedure of IAFM with interference collection mode is shown in Figure 7. When AP transmits a FD-CTS frame to STA1, the FD mode subfield in FD-CTS is interference collection mode to indicate that AP wants to send an ISC frame for interference collection. Thus, STA1 cannot transmit an A-MPDU frame to AP after receiving the FD-CTS frame. Then, AP transmits an ISC frame to STA1 and those candidate STAs for full-duplex link (STA2, STA3 and STA4) as the same as that in the downlink transmission procedure of IAFM with interference collection mode. AP selects STA3 for the full-duplex link, and transmits an A-MPDU to STA1 and announces the AID of STA for STA3 in full-duplex link and the uplink transmitting power for STA1 in FD preamble (FD-preamble is illustrated in Figure 8). It should be noticed that the uplink transmitting power could be controlled both in the downlink and uplink transmission procedure of IAFM with interference collection mode (the details are described in 3.3). STA1 receives the FD-preamble, and then transmits an A-MPDU to AP with the transmitting power indicated in FD-preamble. After the A-MPDU transmission, AP and STA3 transmit BA frames simultaneously. Moreover, the NAV in the RTS frame is shorter than the real transmission procedure since STA1 does not know that AP is going to establish a full-duplex link. Thus, the NAV in the FD-CTS frame should be extended a bit. 


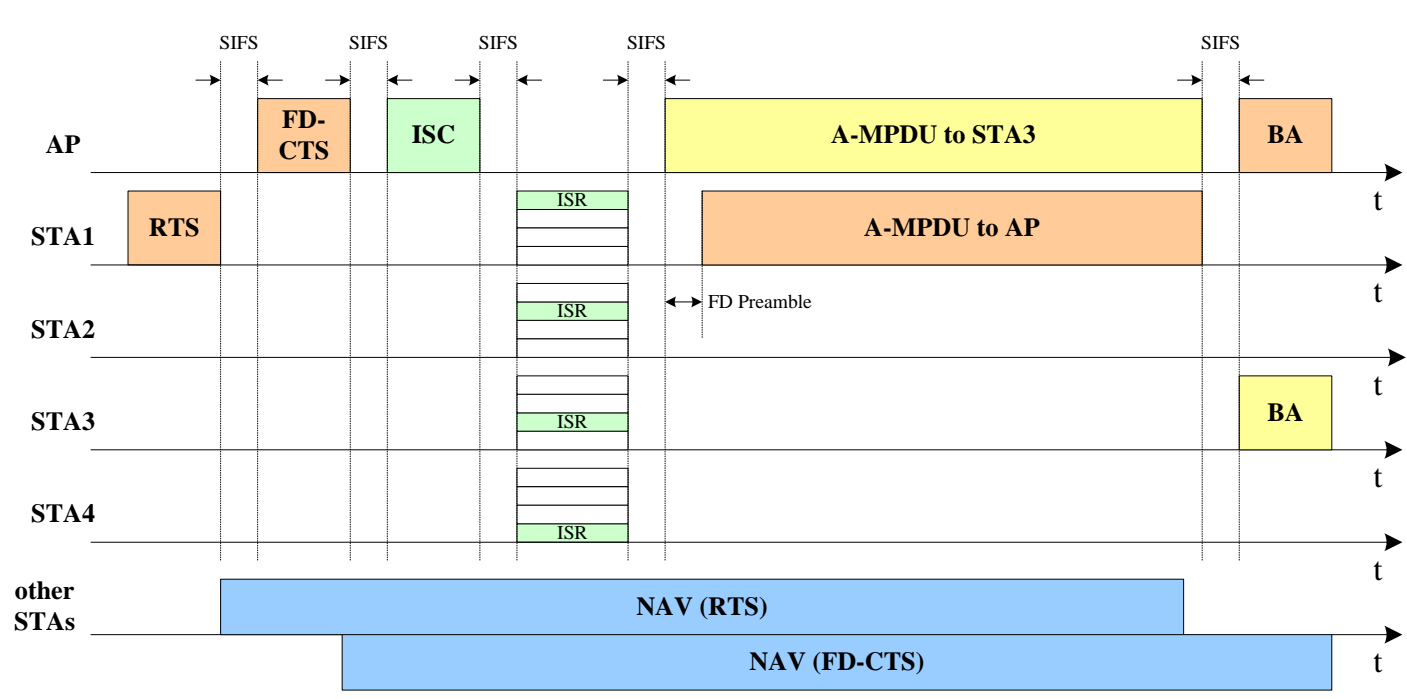

\section{Figure 7. The Uplink Transmission Procedure of IAFM with interference Collection mode}

The format of the ISC frame and the ISR frame and FD-preamble is illustrated in Figure 8. Besides the frame control field, there are $n$ ( $n$ is the number of sub-channels) receiver information field in the ISC frame, and for each receiver information field there are receiver AID sub-field and sub-channel allocation sub-field. In the ISR frame, there are received RTS power sub-field for report the received power of the RTS frame (also for the FD-RTS frame) and received CTS power sub-field for report the received power of the CTS frame (also for the FD-CTS frame). The FD field, which contains FD indicator sub-field, FD AID sub-field and uplink TX power sub-field, is an extra part in FD preamble compared to the normal preamble. The FD indicator sub-field is set to 1 when AP selects a STA for the full-duplex link, and the FD AID sub-field is the AID of the STA in full-duplex link. The uplink TX power sub-field is for the transmitting STA in uplink transmission.

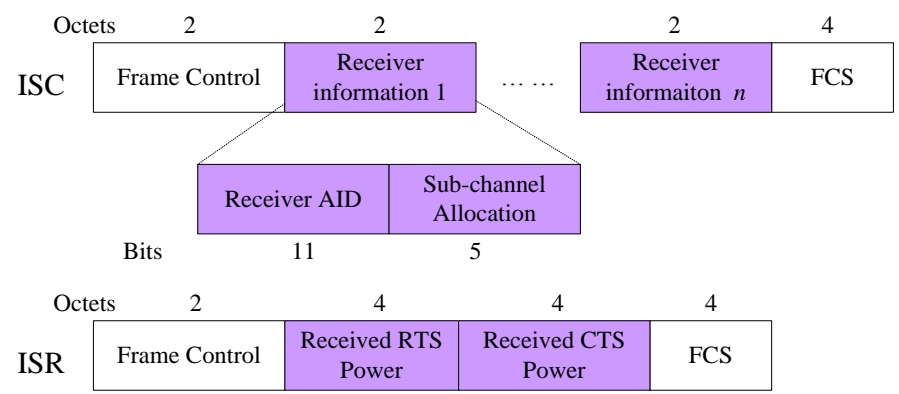

\begin{tabular}{|c|c|c|c|c|}
\hline \multirow[b]{2}{*}{ Preamble } & 8us & 8us & 4us & \\
\hline & $\begin{array}{c}\text { Short Training } \\
\text { Field }\end{array}$ & $\begin{array}{l}\text { Long Training } \\
\text { Field }\end{array}$ & Signal Field & \\
\hline \multirow{3}{*}{ FD-Preamble } & 8us & 8us & 4us & 4us \\
\hline & $\begin{array}{l}\text { Short Training } \\
\text { Field }\end{array}$ & $\begin{array}{l}\text { Long Training } \\
\text { Field }\end{array}$ & Signal Field & FD Field \\
\hline & & $\begin{array}{c}\text { FD Indicator } \\
1 \text { bit }\end{array}$ & $\begin{array}{l}\text { FD AID } \\
11 \text { bits }\end{array}$ & $\begin{array}{c}\text { Uplink TX Power } \\
12 \text { bits }\end{array}$ \\
\hline
\end{tabular}

Figure 8. Frame Format of the ISC and ISR Frame and the FD Preamble 


\subsection{Full-duplex Link Scheduling and Power Control in IAFM}

No matter that AP or STA wins the contention, there are always a uplink A-MPDU frame and a downlink A-MPDU in IAFM when full-duplex link is adopted as shown in Figure 9. On one hand, AP receives the uplink A-MPDU frame from STA2 and suffers from the downlink interference from itself. Since we assume that the self-interference cancellation in AP is perfect, it is quiet easy for AP to receive the uplink A-MPDU frame. On the other hand, STA1 receives the downlink A-MPDU frame from AP and suffers from the uplink interference from STA2, and STA1 may fail to receive the downlink A-MPDU frame due to uplink interference. In other words, the failed transmission only occurs in the STA side. Thus, the most important issue in the selection of STA for the full-duplex link in IAFM is that AP should find a STA which does not cause the failure of the legacy link when AP wins the contention (in Figure 3 and Figure 6) or a STA which does not fail to receive the frame in full-duplex link when STA wins the contention (in Figure 4 and Figure 7).

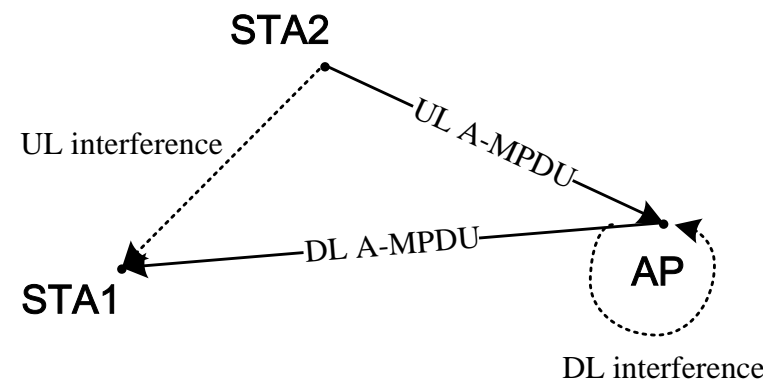

\section{Figure 9. Interference Relationship in IAFM}

Since AP is able to collect the interference state information in interference collection mode (received RTS power and received CTS power sub-field in ISR frame), it is easy for AP to find the proper STA for the full-duplex link. For instance, there are three STA (i.e., STA1, STA2, and STA3) and an AP in a BSS. If AP transmits an A-MPDU frame to STA1 in the legacy link, AP needs to evaluate the signal to interference and noise ratio (SINR) of STA1 under the interference of STA2 (expressed as $\left.S I N R_{1,2}\right)$ and the SINR of STA2 under the interference of STA3(expressed as $S I N R_{1,3}$ ). We assume that the evaluated $S I N R_{1,2}$ is bigger than or equal to the target SINR for STA1 receiving the A-MPDU frame from AP, while $\operatorname{SINR}_{1,3}$ is not. Thus, AP selects STA2 for the full-duplex link. Similarly, if STA1 transmits an A-MPDU frame to AP in the legacy link, AP needs to evaluate the SINR of STA2 under the interference of STA1 (expressed as $S I N R_{2,1}$ ) and the SINR of STA3 under the interference of STA1 (expressed as $S I N R_{3,1}$ ). We assume that the evaluated $\operatorname{SINR}_{2,1}$ is bigger than or equal to the target SINR for STA2 receiving the A-MPDU frame from AP, and $\operatorname{SINR}_{3,1}$ is also bigger than or equal to the target SINR for STA3 receiving the A-MPDU frame from AP. Thus, AP randomly selects one STA for the full-duplex link between STA2 and STA3.

Another factor, which influence the scheduling result of AP in IAFM, is power control. As shown in Figure 10, if the downlink of AP $\rightarrow$ STA1 is the legacy link, the bigger circle is the interference zone with Pt. If any STAs in this circle transmits an AMPDU to AP using the power of $P t$, STA1 cannot receives the A-MPDU from AP successfully. And the smaller circle is the interference zone with $P t / 2$. It is obvious that if the uplink transmission uses less power, there is a better opportunity of establishing the 
full-duplex link. In other words, the power control mechanism is able to improve the performance of IAFM. Thus, power control is an optional mechanism in IAFM.

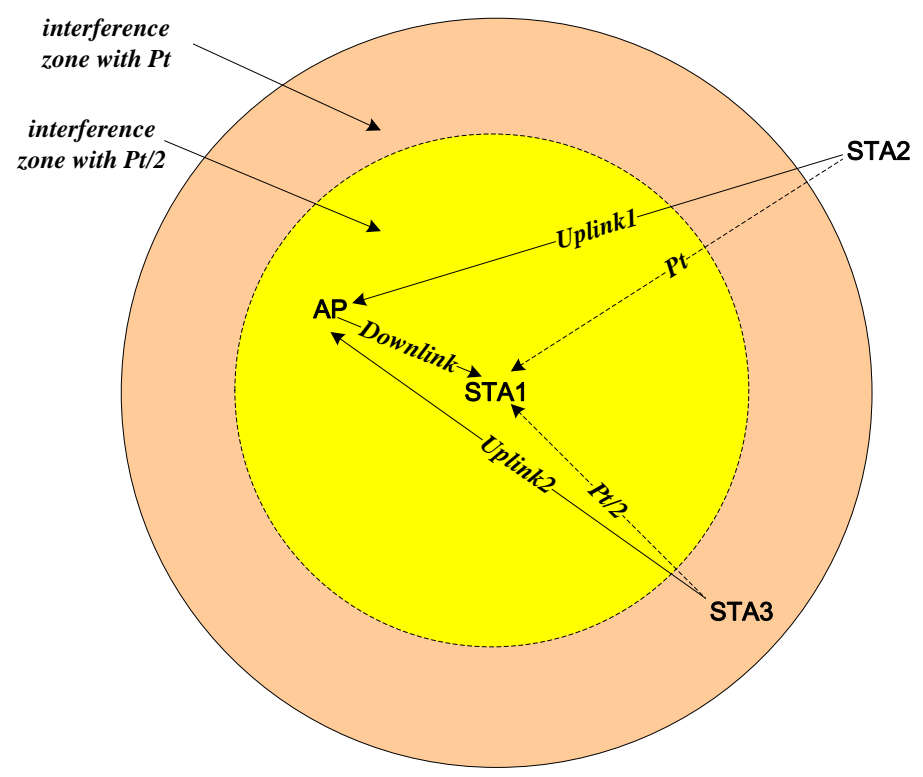

Figure 10. The Interference Zone with Different Transmitting Power in IAFM

\section{Simulation Result}

\subsection{Simulation Parameters}

To evaluate the performance of our proposed IAFM protocol, a simulation platform is built in NS2 [23]. In our simulation, there is only one BSS with an AP and a number of STA around AP. The range of the BSS is $40 \mathrm{~m}$, and AP is in the center of the BSS. The parameter used in simulation is listed in Table 1, and the modulation and coding (MCS) in simulation is listed in Table 2.

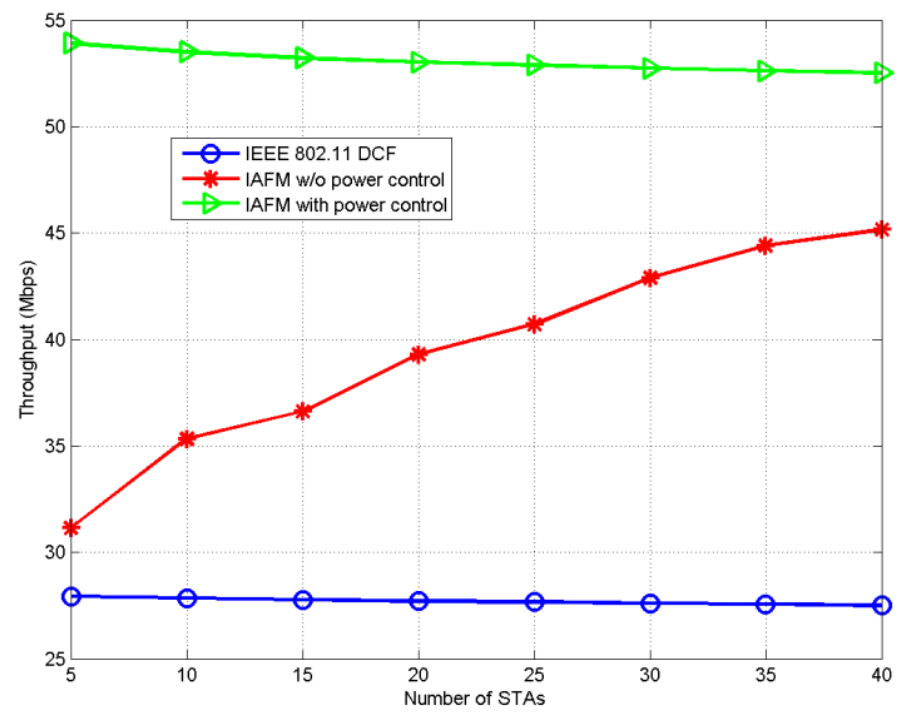


Table 1. The Parameter used in Simulation

\begin{tabular}{|c|c|c|c|}
\hline $\begin{array}{c}\text { Parame- } \\
\text { ters }\end{array}$ & Value & Parameters & Value \\
\hline Preamble & 20us & CTS & $\begin{array}{r}14 \\
\text { Byte }\end{array}$ \\
\hline $\begin{array}{l}\text { FD Pream- } \\
\text { ble }\end{array}$ & $24 u s$ & FD-CTS & $\begin{array}{r}14 \\
\text { Byte }\end{array}$ \\
\hline Payload & $\begin{array}{r}500 \\
\text { Byte }\end{array}$ & BA & $\begin{array}{r}64 \\
\text { Byte } \\
\end{array}$ \\
\hline DIFS & 34us & MAC header & $\begin{array}{c}28 \mathrm{Byt} \\
\mathrm{e}\end{array}$ \\
\hline SIFS & 16us & Delimiter length & 4 Byte \\
\hline Slot time & 9us & $\begin{array}{c}\text { Aggregation } \\
\text { packet }\end{array}$ & 10 \\
\hline RTS & $\begin{array}{r}20 \\
\text { Byte }\end{array}$ & CWmin & 15 \\
\hline FD-RTS & $\begin{array}{r}20 \\
\text { Byte }\end{array}$ & CWmax & $\begin{array}{l}102 \\
3\end{array}$ \\
\hline
\end{tabular}

Table 2. The MCS Adopted in the Simulation

\begin{tabular}{|c|c|c|c|c|}
\hline Mode & Modulation & Coding rate & PHY rate & Target SINR \\
\hline 1 & BPSK & $1 / 2$ & $6 \mathrm{Mbps}$ & $5 \mathrm{~dB}$ \\
\hline 2 & BPSK & $3 / 4$ & $9 \mathrm{Mbps}$ & $6 \mathrm{~dB}$ \\
\hline 3 & QPSK & $1 / 2$ & $12 \mathrm{Mbps}$ & $7 \mathrm{~dB}$ \\
\hline 4 & QPSK & $3 / 4$ & $18 \mathrm{Mbps}$ & $9 \mathrm{~dB}$ \\
\hline 5 & $16-\mathrm{QAM}$ & $1 / 2$ & $24 \mathrm{Mbps}$ & $13 \mathrm{~dB}$ \\
\hline 6 & $16-\mathrm{QAM}$ & $3 / 4$ & $36 \mathrm{Mbps}$ & $17 \mathrm{~dB}$ \\
\hline 7 & 64-QAM & $2 / 3$ & $48 \mathrm{Mbps}$ & $20 \mathrm{~dB}$ \\
\hline 8 & 64-QAM & $3 / 4$ & $54 \mathrm{Mbps}$ & $22 \mathrm{~dB}$ \\
\hline
\end{tabular}

\subsection{Saturation Throughput}

The simulation result of saturation throughput is illustrated in Figure 11 and Figure 12. In Figure 11, the number of STAs is 25 , and the PHY rate is from $6 \mathrm{Mbps}$ to $54 \mathrm{Mbps}$. The saturation throughput of IAFM protocol is always higher than that of DCF, and the saturation throughput of IAFM with power control is almost $200 \%$ of the saturation throughput in DCF. It is obvious that the throughput of IAFM without power control is similar to the throughput of IAFM with power control, but IAFM with power control has advantage in higher PHY rate. Moreover, it is difficult for IAFM protocol with power control to find a proper STA for the full-duplex link when the PHY rate is 54Mbps due to the higher target SINR of $54 \mathrm{Mbps}$ rate. In Figure 12, the PHY rate is $36 \mathrm{Mbps}$, and the number of STAs is from 5 to 40. The IAFM protocol always delivers a higher throughput compared to DCF. And the more STAs is in the networks, the higher throughput of IAFM without power control can achieve. However, the increasing number of STAs just influences IAFM with power control a bit. 


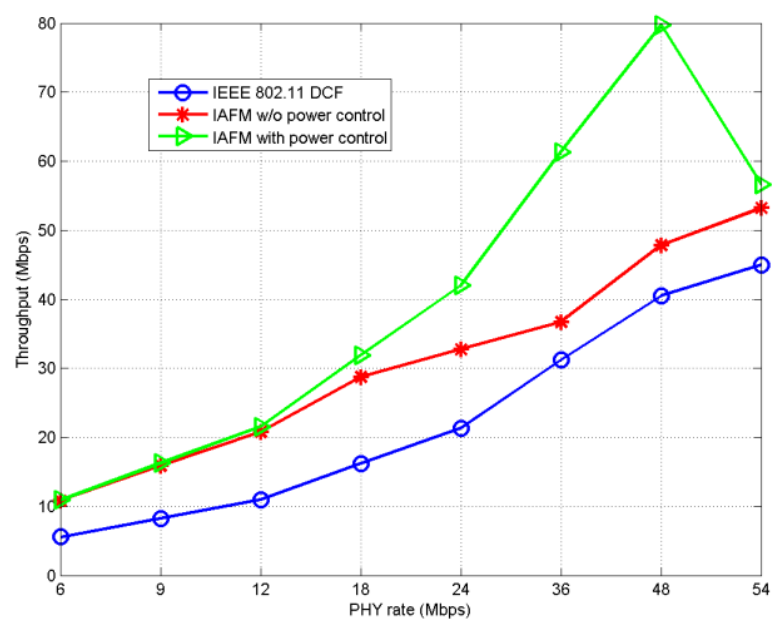

Figure 11. The Throughput with Different PHY Rate

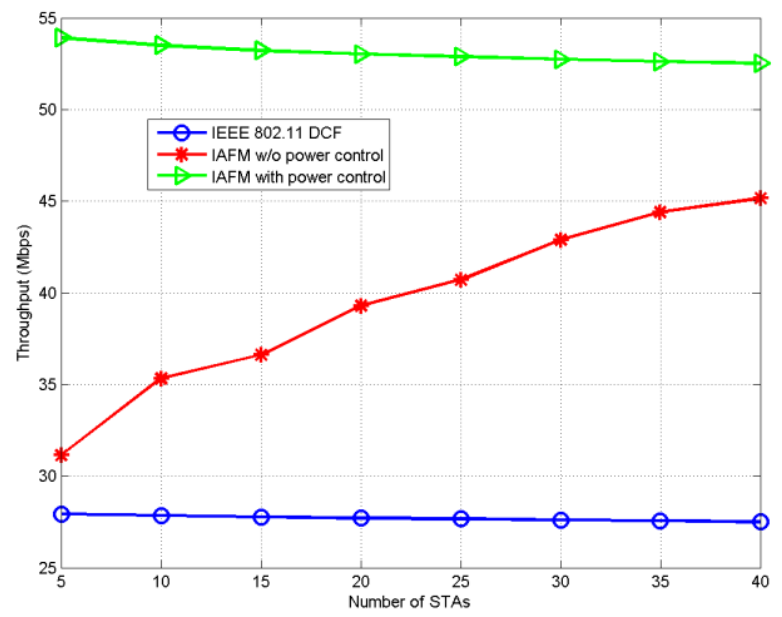

Figure 12. The Throughput with Different STA Number

\subsection{Full Duplex Probability}

The simulation result of the probability of successful full-duplex is illustrated in Figure 13 and Figure 14. The probability of successful full-duplex is defined as the number of establishing the full-duplex link divided by the number of successful channel access. In Figure 13, the probability of successful full-duplex of IAFM without power control decreases severely since the target SINR for receiving frame raises as the PHY rate increases. However, the probability of successful full-duplex of IAFM with power control is not affect by the increasing target SINR except for that in 54Mbps. In Figure 14, it is clear that with the increasing STAs IAFM without power control has more opportunity to establish the full-duplex link, while IAFM with power control can always set up the full-duplex link. 


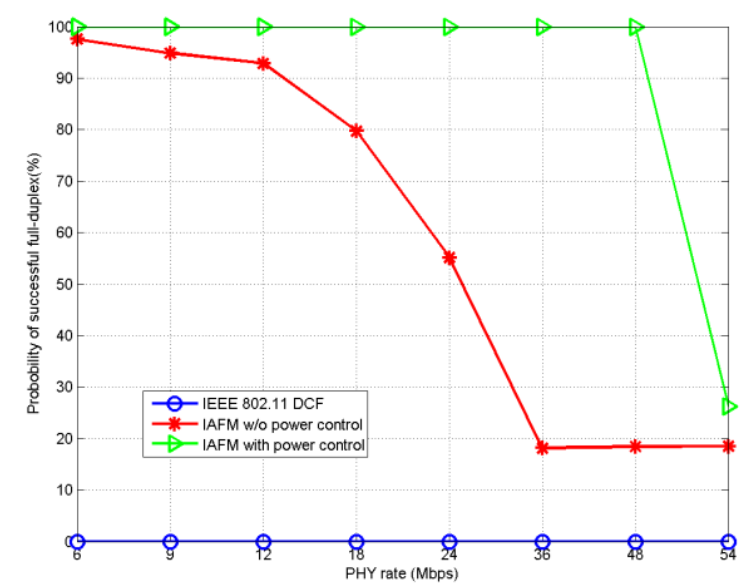

Figure 13. The Probability of Successful Full-Duplex with Different PHY Rate

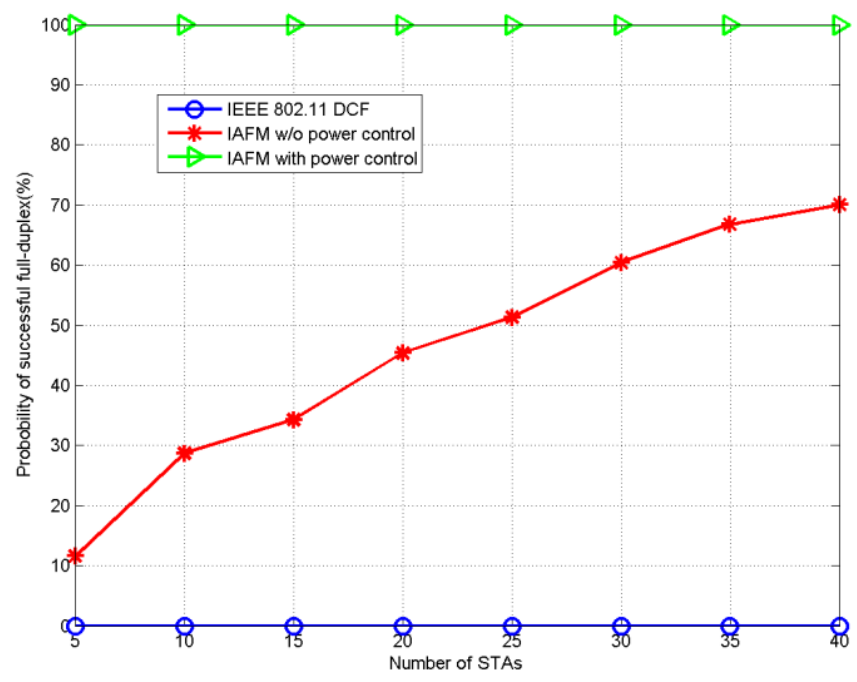

Figure 14. The Probability of Successful Full-Duplex with Different STA Number

\section{Conclusion}

A MAC protocol named as IAFM is proposed in this paper on the assumption that only AP has full-duplex capability. IAFM protocol supports direct scheduling mode, interference collection mode and no full-duplex mode. In direct scheduling mode, AP selects a STA for the full-duplex link and announces the AID of this STA in new designed FDRTS or FD-CTS frame. In interference collection mode, the STAs in the BSS reports their interference state information in OFDMA manner to reduce the overhead. In no full-duplex mode, the nodes including AP and STAs obey DCF protocol in IEEE 802.11. The power control mechanism in IAFM is described and analyzed. The simulation results shows that IAFM with power control improves the throughput greatly compared to the DCF. Thus, IAFM is a flexible and backward compatible full-duplex MAC protocol for the next generation WLANs. 


\section{References}

[1] D. J. Deng, K. C. Chen and R. S. Cheng, “ IEEE 802.11ax: Next generation Wireless Local Area Networks", Proceedings of the 10th International Conference on Heterogeneous Networking for Quality, Reliability, Security and Robustness (QShine), (2014).

[2] “IEEE Std 802.11-2012", IEEE Standard for Information Technology Telecommunications and Information Exchange between Systems Local and Metropolitan Area Networks Specific Requirements Part 11: Wireless LAN Medium Access Control (MAC) and Physical Layer (PHY) Specifications, (2012).

[3] R. Stacey, IEEE 802.11-15/0132r13 Specification Framework for TGax, (2015).

[4] R. Wei, J. Zhen and L. Bao, "Study on Mining Big Users Data in the Development of Hubei Auto-parts Enterprise", Review of computer engineering studies, vol. 2, no. 4, (2015).

[5] M. Duarte, C. Dick and A. Sabharwal, "Experiment-driven characterization of full-duplex wireless systems", IEEE Transactions on Wireless Communication, vol. 11, no. 12, (2012).

[6] M. Duarte, A. Sabharwal, V. Aggarwal, R. Jana, K. K. Ramakrishnan, C. W. Rice and N. K. Shankaranarayanan, "Design and characterization of a full-duplex multiantenna system for WiFi networks", IEEE Transactions on Vehicular Technology, vol. 63, no. 3 (2014).

[7] E. Everett, A. Sahai and A. Sabharwal, "Passive self-interference suppression for full-duplex infrastructure nodes", IEEE Transactions on Wireless Communication, vol. 13, no. 2, (2014).

[8] D. Bharadia, E. McMilin and S. Katti, Editors, "Full duplex radios", Proceedings of the ACM SIGCOMM, (2013).

[9] J. I. Choi, M. Jain, K. Srinivasan, P. Levis and S. Katti, "Achieving Single Channel, Full Duplex Wireless Communication", Proceedings of the 16th Annual International Conference on Mobile Computing and Networking, (2010).

[10] S. S. Hong, J. Mehlman and S. Katti, "Picasso: Flexible RF and spectrum slicing", SIGCOMM Computer Communication, vol. 42, no. 4, (2012).

[11] M. Duarte and A. Sabharwal, "Full-duplex wireless communications using off-the-shelf radios: Feasibility and first results", Proceedings of the Forty Fourth Asilomar Conference on Signals, Systems and Computers, (2010).

[12] A. Sahai, "Wireless full-duplex: From practice to theory", Ph.D. dissertation, Univ. of Rice, Houston, (2014).

[13] A. Sabharwal, P. Schniter, D. Guo, D. W. Bliss, S. Rangarajan and R. Wichman, IEEE Journal on Selected Areas in Communications, vol. 32, no. 9, (2013).

[14] O. A. Magd, IEEE 802.11-14/0165r1 802.11 HEW SG Proposed PAR, (2014).

[15] N. Singh, D. Gunawardena, A. Proutiere, B. Radunovic, H. Balan and P. Key, "Efficient and fair MAC for wireless networks with self-interference cancellation", Proceedings of IEEE WiOpt, (2011).

[16] J. Y. Kim, O. Mashayekhi, H. Qu, M. Kazadiieva and P. Levis, "Janus: A novel MAC protocol for full duplex radio", Stanford Univ. Comput. Sci., Stanford, (2013).

[17] W. Zhou, K. Srinivasan and P. Sinha, "RCTC: Rapid concurrent transmission coordination in full duplex wireless networks", Proceedings of IEEE ICNP, (2013).

[18] A. Sahai, G. Patel and A. Sabharwal, "Pushing the limits of full-duplex: Design and real-time implementation", Rice tech report, (2011)

[19] J. Y. Kim, O. Mashayekhi, H. Qu, M. Kaz and P. Levis, "Janus: A novel mac protocol for full duplex radio", CSTR 2013-02 7/23/13, (2013).

[20] Aimin Tang and Xudong Wang, "A-Duplex: Medium Access Control for Efficient Coexistence between Full-Duplex and Half-Duplex Communications", IEEE Transactions on Wireless Communications, vol. 14, no. 10, (2015).

[21] Qiao Qu, Bo Li, Mao Yang, Zhongjiang Yan, Xiaoya Zuo and Qiaoyan Guan, "FuPlex: A Full Duplex MAC for the Next Generation WLAN", Proceedings of the 11th International Conference on Heterogeneous Networking for Quality, Reliability, Security and Robustness (QShine), (2015).

[22] W. Choi, H. Lim and A. Sabharwal, "Power-Controlled Medium Access Control Protocol for FullDuplex WiFi Networks", IEEE Transactions on Wireless Communications, vol. 14, no. 7, (2015).

[23] The network simulator NS2, http://www.isi.edulnsnam/ns/. 